\title{
Updating the Competency Profile and Examination Blueprint for Entry-Level Optometry in Canada
}

\author{
David Cane, B.Sc., Ph.D \\ Catalysis Consulting
}

Margaret Penny, O.D., M.Ed. Mayfair Eye Care Former Board Chair, Optometry Examining Board of Canada

\section{Anthony Marini, Ph.D}

Martek Assessments Ltd.

Tami Hynes, M.B.A. ${ }^{\mathrm{i}}$

Chief Executive Officer, Optometry Examining

Board of Canada

\begin{abstract}
The Optometry Examining Board of Canada (formerly Canadian Examiners in Optometry) is a not-for-profit corporation that administers the entry-topractice examination for optometrists in Canada in service to its members, the 10 provincial optometry regulators.
\end{abstract}

The described work resulted in an updated entry-level competency profile for optometry, together with an examination blueprint based upon indicators derived from the competencies. The project took place over the period May 2014 - September 2015 and involved the following steps:

- Establishment of project teams

- Clarification of conceptual framework

- Development of proposed competencies

- Validation of competencies

- Development of indicators for each competency, consistent with the assessment methodologies used in the entry-to-practice examination

- Construction of an updated examination blueprint

SUGGESTED KEY WORDS

Assessment, competence, competency-based standards, entry-topractice (ETP), examination blueprint, objective structured clinical examination (OSCE), optometrist, registration (licensure) examination 


\section{INTRODUCTION}

The Optometry Examining Board of Canada (OEBC; formerly Canadian Examiners in Optometry, CEO) was established in 1995 through collaboration among Canadian provincial optometry regulatory bodies. OEBC is a not-for-profit corporation with a mandate to develop and administer a national entry-to-practice (ETP) examination for optometrists. The examination has been offered since 1995, the first examinations having been developed with input from members of the profession following a review of existing entry-level examinations and education in optometry. Success in the examination is required for registration as an optometrist in most provinces within Canada.

Since the mid-1990s, competency-based ETP examinations have become the gold standard in Canada. This expectation was formalized on a national level with the introduction by the Government of Canada of the Agreement on Internal Trade in $1994^{1}$ which noted:

"Any measure adopted or maintained by a regulatory body or government relating to occupational licensing ... should relate principally to competence;" and

“Competencies can be acquired through different combinations of training and experience”

The use of practice-based competencies as requirements for registration has two important advantages: it ensures that ETP requirements are in the public interest, and it fosters accountability among practitioners.

Ideally the blueprint ${ }^{\mathrm{ii}}$ of an ETP examination is derived from an ETP competency profile, and the curriculum of education programs that serve as pre-registration requirements is directly related to the same profile.

In 2001, OEBC formed a Competence Committee that worked over a span of four years to develop CompetencyBased Performance Standards (2005)., ${ }^{2,3}$ The work was grounded on the World Health Organization's survey identifying common competencies across the health professions, and similar efforts of the National Association of Pharmacy Regulatory Authorities and the Australian Office of Education.

The 2005 Standards were based on four competency roles: provide comprehensive eye and vision care; collaborate; manage; and educate. They also identified "general attributes of professional competencies."

In August 2015, OEBC published updated competency-based requirements for entry to the profession, entitled National Competency Profile for Entry-Level Optometry. ${ }^{4}$ The 2015 profile is based on an explicit conceptual framework that describes the relationship between the competencies and professional competence, and includes a definition of entry-level proficiency. OEBC followed up with the creation of a new examination blueprint based upon the 2015 profile, which led to the implementation of a new ETP examination in May 2017. This report describes the development and structure of the 2015 competency profile and the resulting examination blueprint.

THE PROJECT

In May 2014, the Board of Directors of OEBC approved a project to develop an updated national competency profile and examination blueprint. The project was announced through letters to members and stakeholders, including OEBC volunteers.

The project began that same month, with the identification of key personnel. A consultant in competency-based standards was engaged to lead competency development and validation, and indicator development. OEBC's psychometric consultant was charged with leading development of the examination blueprints.

Content expertise for competency and indicator development was provided by a 4-person subject matter expert team working under the guidance of the consultant. Team members were selected from volunteers, based on pre-established criteria. Three team members were experienced optometrists in general practice and one was a senior optometry educator with a Canadian university program. All members had extensive familiarity with entry-level optometry practice and some had previously participated in OEBC's examination development process. None were currently serving in any capacity with the OEBC or any other assessments of entry-level competence in optometry. 
Development of the competencies and indicators took place from July 2014 - May 2015. The bulk of the work was completed within approximately 75 hours of team meeting time, comprised of both in-person and online sessions, supplemented by individual study and one-on-one discussions.

Status reports were provided to OEBC by the consultant throughout the project. The new competency profile was approved by OEBC and published on its website in August 2015.

Subject matter expertise for blueprint development and new examination design was provided by a 6-person team working under the guidance of the psychometric consultant. The team consisted of experienced optometrists who had worked for several years supporting OEBC's examination.

Blueprint development and examination design took place over the period June - September 2015. The bulk of the team's work was completed in 3 in-person meetings and 3 teleconferences totalling approximately 40 hours of meeting time. The new blueprints were approved by OEBC and published on its website. ${ }^{5}$

\section{CONCEPTUAL FRAMEWORK}

\section{Competence and Competencies}

The term competence is generally understood to refer to effective performance. Competence is a concept widely referred to in the professional literature, and was defined by Kane in 1992 as "the degree to which an individual can use the knowledge, skills and judgements associated with the profession to perform effectively within the domain of professional encounters defining the scope of professional practice.” ${ }^{6}$ In 2002, Epstein and Hundert noted that "competence is developmental, impermanent and context-specific." More recently (2015) the Royal Society of Physicians and Surgeons of Canada observed "[physician] competence is both conditional on, and constrained by, ... practice context, is dynamic and continually changes over time."

We use competence to mean effective workplace performance in the context and in the moment of practice.

Other terms related to competence (for example "competency", "a competency", "competencies", "competent") are also in common usage although their meanings are not standardized. Occupational competency profiles (lists of competencies required for effective performance) have been developed and published by many professions, but no common framework or set of definitions has been established.

It is often stated that McClelland originated the occupational competency movement in 1973 when he suggested that successful workplace performance depends more upon a worker's specific knowledge, abilities and attributes than upon his or her intelligence. ${ }^{9}$ Concepts of competence have since become a driving force in development of specifications for the workplace and for education. In 1992 the Office of Personnel Management of the United States Government defined a competency as "a measurable pattern of knowledge, skills, abilities, behaviors, and other characteristics that an individual needs to perform work roles or occupational functions successfully". ${ }^{10}$ There have since been many variations on this understanding of competencies, including some which have considered competencies to be the knowledge, skills and attributes required to perform in the workplace, while others have considered competencies to be the workplace outcomes that result from the application of knowledge, skills and attributes.

More recent attention has focused on competencies as abilities. Kaslow and co-workers (2007) noted that "it is essential that competencies be conceptualized as generic, wholistic (sic) and developmental abilities." ${ }^{\prime 1}$ The Royal Society of Physicians and Surgeons of Canada's CanMEDS 2015 defines a competency as "an observable ability of a health professional, integrating multiple components such as knowledge, skills, values and attitudes." 12

We think of competencies as the micro-level abilities that enable competent (macro-level) performance in the practice context. In brief, we say that competencies enable competence.

We define a competency as the ability to perform a specific workplace task with a prescribed level of proficiency.

As noted above, competence is developmental, impermanent and context-specific. The competency sets that enable competence continually evolve over the span of a professional's career, as the result of ongoing informal and formal learning. The evolution of occupational abilities over time was recognized by Dreyfus and Dreyfus in $1986^{13}$ and the Dreyfus developmental model has since been applied to professions including nursing ${ }^{14}$ and medicine ${ }^{15}$, as well as optometry. ${ }^{16}$ 


\section{Entry-Level Proficiency}

Since one of the principal uses of an ETP competency profile is the assessment of ETP competence, competencies must specify not just the workplace tasks that can be performed but also the level of performance expected and any related contextual constraints. These factors are sometimes referred to as standards and conditions for task performance. In our work, we use the term level of proficiency to refer to standards and conditions applicable to competencies.

At ETP, we expect a practitioner to demonstrate in all tasks, as a minimum, entry-level proficiency, which we characterize as follows:

Entry-level proficiency involves addressing common patient presentations, and critical patient presentations, independently, within an appropriate time frame, and achieving outcomes consistent with the generally-accepted standards of the profession; this includes the ability to recognize complex situations that are beyond the capacity of the entry-level optometrist, and addressing them by seeking advice or consultation, by reviewing research literature, and/or by referral to a more experienced optometrist or a more appropriate health care professional.

\section{Assessment of Competence}

A variety of methodologies are used in attempts to assess competence in regulated professions. ${ }^{17}$ In Canadian healthcare, the most common ETP assessment methods are written testing (typically multiple choice questions, MCQ) and performance-based testing through simulation (objective structured clinical examination, OSCE). OEBC's examination uses both written and performance-based assessments.

Since competencies are abilities to perform in the workplace (i.e., practice), ideally, assessment should take place in a practice setting. However, for reasons of practicality and standardization, most ETP assessments are administered prior to registration and take place not in practice but in specifically constructed assessment vehicles (such as casebased written examinations and OSCE stations).

In recognition of this distinction, we use the term "indicator" to refer to a behaviour that is observable within a specific assessment methodology and which provides an indication that the candidate possesses a competency.

Consistent with the conceptual framework we describe above, the project proceeded stepwise as follows:

- Step 1: Development and validation of competencies (each described as a practice ability)

- Step 2: Identification of potential indicator(s) for each competency (each described as an observable derived from a competency and consistent with the constraints of OEBC's assessment methodologies)

- Step 3: Development of the examination blueprint derived from the validated competencies and their indicators

\section{DEVELOPMENT OF PROPOSED COMPETENCIES}

To enhance the readability of the competency profile and its utility for broad audiences both within and outside of the profession, a simple functional-based structural framework was identified (Table 1), consisting of nine areas of optometry practice. Functional frameworks are commonly (but not universally) used for the organization of competencies. They have the advantages of being readily understandable to diverse users of the competency profile, and, from the perspective of the team developing the competencies, facilitating comprehensive and balanced coverage of all areas of practice.

It was further agreed that each competency statement would describe a single, stand-alone practice task, to facilitate indicator and test item development. It is important to recognize that, in optometry practice, tasks are not undertaken in isolation; the competencies in the profile must be seen as an integrated set of abilities, with each informing and qualifying the others.

An initial draft of competencies was developed by drawing content from OEBC's 2005 Competency-Based Performance Standards, organizing it within the 9 practice areas, and adjusting consistent with the "one task per 
statement" rule described above. The team then studied a variety of published materials describing standards for optometry practice and education, both in North America and internationally. These materials were used both to confirm that the content of the emerging competency profile was in general equivalent to established international standards, and to suggest entry-level expectations that may be missing from the initial draft.

Principal reference documents are listed in Table 2.

Table 1: Nine Areas of Optometry Practice

\begin{tabular}{|l|l|}
\hline A1 & Communication \\
\hline A2 & Professionalism \\
\hline A3 & Patient-Centered Care \\
\hline A4 & Assessment \\
\hline A5 & Diagnosis and Planning \\
\hline A6 & Patient Management \\
\hline A7 & Collaborative Practice \\
\hline A8 & Scholarship \\
\hline A9 & Practice Management \\
\hline
\end{tabular}

Table 2: Principal Reference Documents

\begin{tabular}{|l|l|}
\hline WCO Competency Model (2013) & $\begin{array}{l}\text { WCO Headquarters, 243 North Lindbergh Blvd., St Louis, } \\
\text { MO 63141-7881, USA; } \\
\text { http://www.worldoptometry.org }\end{array}$ \\
\hline $\begin{array}{l}\text { Accreditation Manual: Professional Optometric Degree } \\
\text { Programs (2013) }\end{array}$ & $\begin{array}{l}\text { Accreditation Council on Optometric Education, 243 North } \\
\text { Lindbergh Blvd., St. Louis, MO 63141 USA; www.theacoe.org }\end{array}$ \\
\hline Universal and Therapeutic Competency Standards (2008) & $\begin{array}{l}\text { Optometrists Association Australia; } \\
\text { http://www.optometry.org.au }\end{array}$ \\
\hline $\begin{array}{l}\text { Optometry Core Curriculum, Core Competencies and } \\
\text { Learning Outcomes (2010) }\end{array}$ & $\begin{array}{l}\text { General Optical Council, 10 Old Bailey, London EC4M 7NG, UK; } \\
\text { www.optical.org }\end{array}$ \\
\hline $\begin{array}{l}\text { Draft CanMEDS 2015 Physician Competency Framework - } \\
\text { Series I (2014) }\end{array}$ & $\begin{array}{l}\text { Royal College of Physicians and Surgeons of Canada, 774 } \\
\text { Echo Drive, Ottawa, ON K1S 5N8, Canada; } \\
\text { www.royalcollege.ca }\end{array}$ \\
\hline & $\begin{array}{l}\text { National Board of Examiners in Optometry, 200 South } \\
\text { College Street, \#2010, Charlotte, NC 28202, USA; } \\
\text { www.optometry.org }\end{array}$ \\
\hline
\end{tabular}

Following a review for comprehensiveness and balance of content across all practice areas, and to ensure consistent language and style, the initial product consisted of 101 proposed competency statements. The competencies were translated into French by a registered professional translator working with a bilingual optometrist.

PRACTICE-BASED VALIDATION OF COMPETENCIES

A bilingual (English-French) online survey was used to obtain feedback on the proposed competencies from practicing optometrists across Canada. Survey invitations were sent to all registrants by provincial regulatory bodies. To maximize the number of responses, respondents were offered continuing education credits by their respective regulator (Quebec excluded) for completing the survey. 
Demographic questions in the survey identified the primary province of practice and the nature, extent and currency of practice experience.

Survey completers were asked to rate their response to three questions about each competency:

- In the context of providing safe, effective and ethical patient care in your optometric practice, how important is the performance of this [competency]? (Rate on a scale of very important / important / somewhat important / not important (or not relevant in my practice)). We refer to this as the "importance rating".

- How frequently do you personally perform this [competency]? (Rate on a scale of very frequently / frequently / occasionally / rarely / never). We refer to this as the "frequency rating".

- In your opinion should proficiency in this [competency] be an expectation of optometrists at the point of entry-to-practice? (Rate on a scale of yes / no / not sure). We refer to this as the "ETP rating".

In addition to the rating questions, respondents were invited to suggest competencies that they considered to be realistic expectations of optometrists at entry-to-practice but were missing from the list.

OEBC sent a link to access the survey to the provincial optometry regulators who invited all their registrants to respond. The survey was open for four weeks in January 2015 and received 1,185 complete responses (representing $23 \%$ of O.D.s nationally). The sample size produced a margin of error for numerical conclusions drawn from the survey of better than $+/-3 \%$ at $95 \%$ confidence.

The number of responses by province is provided in Table 3.

Table 3: Survey Responses by Province

\begin{tabular}{|l|l|l|l|}
\hline Province & $\begin{array}{l}\text { Approximate } \\
\text { O.D. population } \\
\text { (2015) }\end{array}$ & $\begin{array}{l}\text { Survey } \\
\text { responses }\end{array}$ & $\begin{array}{l}\text { \% O.D.s } \\
\text { responding }\end{array}$ \\
\hline BC & 680 & 167 & 25 \\
\hline AB & 645 & 115 & 18 \\
\hline SK & 156 & 55 & 35 \\
\hline MB & 148 & 41 & 28 \\
\hline ON & 1,939 & 508 & 26 \\
\hline QC & 1,353 & 200 & 15 \\
\hline NB & 118 & 52 & 44 \\
\hline NS & 118 & 34 & 29 \\
\hline PE & 20 & 8 & 40 \\
\hline NL & 61 & 5 & 8 \\
\hline Canada & 5,238 & 1,185 & 23 \\
\hline
\end{tabular}

Importance and frequency response data for each competency were ranked as High, Medium or Low based upon the proportion of respondents who selected the two highest ratings ("very important" / "important"; "very frequent" / "frequent", respectively). The following ranking criteria were used: 
- If more than two-thirds of the respondents selected one of the 2 highest ratings, then rank = High

- If between one-third and two-thirds of the respondents selected one of the 2 highest ratings, then rank = Medium

- If less than one-third of the respondents selected one of the 2 highest ratings, rank = Low

Response data for the question as to whether the competency should be an ETP expectation was ranked as High, Medium or Low based upon the proportion of respondents who selected "yes", after first eliminating "not sure" ratings. The following criteria were used:

- If more than two-thirds of the respondents selected "yes," then rank = High

- If between one-third and two-thirds of the respondents selected "yes," then rank = Medium

- If less than one-third of the respondents selected "yes," then rank = Low

An analysis of survey data was undertaken to determine whether there were significant geographical variations in the responses. Table 4 breaks down the survey participation geographically. Provincial ratings were grouped into five regions to address the issue of small provincial sample sizes. The regional grouping provides a more robust basis for conducting data analysis and facilitates the interpretation of analysis results.

Table 4: Survey Responses by Region

\begin{tabular}{|l|l|l|l|}
\hline Province(s) & Region & Frequency & Percent \\
\hline BC & British Columbia & 167 & 14 \\
\hline AB, SK, MB & Prairie & 211 & 18 \\
\hline ON & Ontario & 508 & 43 \\
\hline QC & Quebec & 200 & 17 \\
\hline NB, NS, PE, NL & Atlantic & 99 & 8 \\
\hline & Canada & 1,185 & 100 \\
\hline
\end{tabular}

We conducted analysis of variance (ANOVA) tests between regions with respect to both the importance ratings and the frequency ratings within each of the nine practice areas. Overall, there was a high degree of consistency across the regions. The practice area with the largest variance was A9 (Practice Management), with 8\% variability for both importance and frequency. The area with the smallest variance was A8 (Scholarship) with less than 1\% variability for both importance and frequency.

We concluded was that since we draw only broad, qualitative conclusions from survey data (by ranking the level of support for each competency as High, Medium or Low in each rating category), there was no value to be gained by proceeding in our analysis on other than a Canada-wide basis.

In summary, for over 101 proposed competences included in the survey, the rankings were as follows:

- 95 competencies were ranked High for importance (94\%)

- 92 competencies were ranked High as an ETP expectation (91\%)

- 73 competencies were ranked High for all of importance, frequency and ETP expectation (72\%) $)^{\mathrm{iii}}$ 
Each competency statement that received a Medium or Low ranking for any one of importance, frequency or ETP expectation was discussed by the team with the objective of deciding whether it should be retained unchanged in the profile, retained in a modified form, or removed. On this basis, two competencies were eliminated:

\section{Provide patient with information on relevant social support services.}

Use community health care resources and delivery systems to improve care.

In addition, discussions resulted in several wording clarifications and refinements.

The open question that invited respondents to suggest additional ETP competencies drew 138 comments (representing $12 \%$ of respondents). Many comments referred to competencies that were already included in the profile, with respondents emphasizing their importance and sometimes mentioning that they had been insufficiently emphasized in their own education, while other comments referred to optometry education and regulation in general. All open responses were discussed thoroughly by the team, and several resulted in wording adjustments to competency statements. No competencies were added.

At the conclusion of the survey analysis and review, there were 99 competencies remaining in the profile. Of these, 94\% had been ranked High for importance and 92\% were High for ETP expectation (the remainder were ranked Medium). Together with the high degree of consistency in ratings received from across the country, this constitutes a very high degree of validation for the competencies relative to entry-level optometry practice.

\section{DEVELOPMENT OF INDICATORS}

The competency development team proceeded to analyze each of the 99 validated competencies to identify related behaviours that would be potentially observable within the constraints of the two assessment methodologies, MCQ and OSCE. This level of analysis resulted in some further minor adjustments to the competencies themselves: several were viewed as overlapping and were combined, others were re-worded and used as indicators.

The product at the completion of this phase of work was a validated national competency profile with a matrix of 92 competencies and over 300 related indicators. The profile was presented in a workshop in April 2015 to representatives of OEBC's 10 members, Canadian optometry schools, and the Canadian Association of Optometrists.

\section{CONSTRUCTION OF THE EXAMINATION BLUEPRINT}

Following approval of the competency profile by the Board of Directors of OEBC in May 2015, the blueprint team commenced its work.

Kane and colleagues ${ }^{18}$ have provided an approach to determine the relative contributions of specific practice activities to patient care and outcomes. Their method combines frequency and importance ratings for competencies to obtain an overall index reflecting the contribution that each competency makes to ensuring safe and effective entrylevel practice. This approach is commonly used to guide the development of examination blueprints from survey data, and was utilized in our work.

The results of an initial analysis of survey responses using Kane's methodology are reported in Table 5. The nine practice areas together reflect a total of 92 competencies, with the strongest being Assessment.

Blueprint team members reviewed the practice areas and their weightings, the competencies and the indicators, and established the feasibility of their representation in one or both of the assessment methodologies, MCQ and OSCE. Time and setting requirements were considered, with the objective of establishing a fair and defensible examination addressing the fundamental mandate of protecting the public. The team worked by consensus.

Table 6 represents the results of this analysis and the final weightings for the examinations across the 9 practice areas. The new examination is structured to include all practice areas together with 55 competencies and their corresponding performance indicators. Consistent with the initial survey results, A4 Assessment is a dominant focus in the examination. 
Within the written examination, there are 62 cases, each with four multiple-choice questions, reflecting all 9 practice areas. Within the OSCE, there are 16 stations covering six practice areas (Collaborative Practice, Scholarship and Practice Management are not included).

The final blueprint for the written and practical examinations was published on the OEBC website in May 2016.

Table 5: Kane’s Weightings for Practice Areas Based on Survey Data

\begin{tabular}{|l|l|l|}
\hline Practice Area & Weighting & $\begin{array}{l}\text { No. of } \\
\text { Items }\end{array}$ \\
\hline A4 Assessment & 16.2 & 14 \\
\hline A2 Professionalism & 16.2 & 13 \\
\hline A9 Practice management & 16.0 & 16 \\
\hline A6 Patient management & 11.5 & 11 \\
\hline A3 Patient centered care & 9.9 & 9 \\
\hline A5 Diagnosis \& planning & 8.8 & 7 \\
\hline A8 Scholarship & 8.7 & 9 \\
\hline A1 Communication & 6.5 & 6 \\
\hline A7 Collaborative practice & 6.4 & 7 \\
\hline Total & 100.0 & 92 \\
\hline
\end{tabular}

Table 6: Final Weighting and Competency Elements for the Examination

\begin{tabular}{|l|l|l|}
\hline Practice Area & $\begin{array}{l}\text { Number of } \\
\text { Competencies }\end{array}$ & $\begin{array}{l}\text { Weighting } \\
(\%)\end{array}$ \\
\hline A4 Assessment & 12 & 22.8 \\
\hline A6 Patient Management & 10 & 17.7 \\
\hline A3 Patient Centered Care & 8 & 14.6 \\
\hline A5 Diagnosis \& Planning & 7 & 13.8 \\
\hline A1 Communication & 5 & 8.9 \\
\hline A2 Professionalism & 4 & 8.0 \\
\hline A7 Collaborative Practice & 4 & 6.1 \\
\hline A9 Practice Management & 3 & 5.2 \\
\hline A8 Scholarship & 2 & 2.9 \\
\hline Total & 55 & 100.0 \\
\hline
\end{tabular}

IMPLEMENTATION OF THE NEW EXAMINATION

Following the Board of Directors' approval of the blueprint in October 2015, OEBC began work to update the design and content of the examination. The examination continues to use written and performance-based formats and is offered in English and French. 
The format of the written examination is unchanged; the content has been updated to align with the new blueprint. The format of the practical examination has changed from solely skills-based assessment to OSCE, which integrates clinical knowledge, optometric skills, professional judgement and communication; the content has been updated to align with the new blueprint.

Development of the OSCE format required establishing a case-writing team to develop examination content, and integrating OSCE case score-setting into the examination development process. In May 2016, OEBC announced the new ETP examination beginning in May 2017.

\section{Acknowledgements}

The authors wish to thank OEBC for its commitment to developing an updated national competency profile and examination blueprint for entry-to-practice optometry in Canada.

In keeping with OEBC's philosophy to involve practicing optometrists in the development of examination standards, the work of the following optometrists who served as members of the project teams is gratefully acknowledged.

J. Arnel, O.D.; J. Barbour O.D.; K. Hawkes, O.D.; C. Ho, O.D.; P. Hrynchak, O.D.; T. McNab, O.D.; L. Myshak, O.D.; P. Padfield, O.D.; G. Raby, O.D.; P. Rose O.D.

Finally the authors wish to thank the 1,185 optometrists from across Canada who took the time to respond thoughtfully to the competency validation survey and thereby ensured currency in the entry-to-practice requirements for the profession.

\section{ENDNOTES}

i Correspondence should be sent to Tami Hynes, tami.hynes@oebc.ca

ii An examination blueprint specifies the structure and content of an examination and relates the content to a foundational document such as a competency profile. The blueprint provides for a transparent examination process and allows for the examining entity to construct examination forms which, over time, are consistent in validity and reliability.

iii With respect to frequency, it should be noted that ratings of less than High are to be expected for many important competencies, for example: Provide first aid

\section{REFERENCES}

1. Government of Canada. Agreement on Internal Trade, Labour Mobility Chapter. 1994

2. Canadian Examiners in Optometry. Competency-Based Performance Standards for the Canadian Assessment of Competency in Optometry. 2005

3. Winslade T, Winslade N, Chou R, et al. Canadian Journal of Optometry. 2007; Vol 69, No 4: 145-149, No 5: 183-187

4. Canadian Examiners in Optometry. National Competency Profile for Entry-Level Optometry. May 2015. Retrieved May 262017 from http://www.oebc.ca/clientuploads/National\%20Competency\%20 Profile/CEO-ECO\%20National\%20Competency\%20Profile\%20 in\%20Optometry_English.pdf

5. Canadian Examiners in Optometry. OEBC Exam Blueprint - Written \& OSCE. May 2016. Retrieved May 262017 from http://www.oebc. ca/clientuploads/New\%20Exam\%202017/Blueprint\%20Written\%20+\%20OSCE\%20ENG\%20(website).pdf

6. Kane MT. The Assessment of Professional Competence, Evaluation \& The Health Professions. Sage Publications Inc. 1992; Vol 15, No 2: 163-182

7. Epstein RM, Hundert EM. Defining and Assessing Professional Competence. JAMA. 2002; 287: 226-235

8. Royal Society of Physicians and Surgeons of Canada. CanMEDS: Better standards, better physicians, better care. 2015; retrieved September 162016 from http://www.royalcollege.ca/rcsite/canmeds/ canmeds-framework-e

9. McClelland D. Testing for competence rather than for intelligence. American Psychologist. 1973; 28: 1-14
10. United States Government Office of Personnel Management. Retrieved September 162016 from https://www.opm.gov/policy-dataoversight/assessment-and-selection/competencies/

11. Kaslow NJ, Bebeau MJ, Lichtenberg JW, et al. Guiding principles and recommendations for the assessment of competence. Professional Psychology: Research and Practice. 2007; Vol 38, No 5, 443

12. Frank JR, Snell L, Sherbino J, editors. CanMEDS 2015 Physician Competency Framework. 2015. Royal College of Physicians and Surgeons of Canada

13. Dreyfus HL, Dreyfus SE. Mind over machine: The power of human intuition and expertise in the era of the computer. 1986. New York: The Free Press

14. Benner P. From novice to expert: Excellence and power in clinical nursing practice. 1984; Menlo Park: Addison-Wesley

15. Carraccio CL, Benson BJ, Nixon LJ, et al. From the educational bench to the clinical bedside: translating the Dreyfus developmental model to the learning of clinical skills. Acad Med. 2008; Vol 83, No 8, 761-767

16. Faucher C. Development of professional expertise in optometry. Optometry. 2011; 82: 218-223

17. Austin Z, Gregory P, Young L. Professional quality assurance and competency assessment - a scoping review. 2016; College of Physiotherapists of Ontario

18. Kane MT, Kingsbury C, Colton D, Estes C. Combining Data on Criticality and Frequency in Developing Test Plans for Licensure and Certification Examinations. Journal of Educational Measurement. Vol. 26, No. 1 (Spring, 1989), 17-27 